\title{
Analytical Learning Guided by Empirical Technology: An Approach to Integration (Extended Abstract)
}

\author{
Nikolay I. Nikolaev ${ }^{1}$ and Evgueni N. Smirnov ${ }^{2}$ \\ 1 Department of Computer Science, American University in Bulgaria \\ Blagoevgrad 2700, Bulgaria, e-mail: nikolaev@nws.aubg.bg \\ 2 Institute for Information Technologies, Bulgarian Academy of Sciences \\ Acad.G.Bonchev Str.,blok 29A,Sofia 1113,Bulgaria,e-mail: smirnov@iinf.bg
}

\begin{abstract}
An approach to integration of the analytical and empirical learning strategies for solving the theory-based concept specialization problem is proposed. The emphasis is on learning correct concepts within conjunctive description language isolated from supplied domain theory. In order to overcome expressive limitations of such language, the analytical learning component is guided by a specific empirical version space technology. The approach learns incrementally a correct pure conjunctive or DNF concept definition in dependance on the domain theory.
\end{abstract}

\section{Introduction}

The theory-based concept specialization problem (TBCS), defined in [1] and [5], requires a more general formulation for practical applications. There are given: (1) a first order domain theory $D T$ from DNF rules with consequents from one predicate; (2) a goal concept $G C$ defined by the domain theory; (3) a finite set $T S$ of examples described in pure conjunctive first order language, which are positive examples of the concept $G C$, and are positive and negative examples of an unknown concept under study $S C$ ( $S C$ is specialization of $G C$ ). The task is to find a complete and consistent definition of the concept under study $S C$, described in DNF first order language, that is implied by the positive and not by the negative examples in context of the domain theory.

Most of the previous works deal only with specific cases of the above TBCS problem and solve these cases by integrated empirical-after-analytical learning approaches: IOE [1], MECEA [5]. They produce a complete and consistent pure conjunctive concept definition of $S C$ when the domain theory determines a sufficiently expressive concept description language. When the language expressivenes is insufficient (i.e., the definition of $S C$ is DNF ), these approaches fail. This situation arises when in the process of incremental learning either of the two anomalies occurs [4]; (1) incompleteness: a positive example is encountered, but the current concept definitions cannot be generalized to admit it without admitting negative training; (2) inconsistency: a negative example is encountered, but the current concept definitions cannot be specialized to reject it without also rejecting positive training. 
This paper proposes an improved approach, called Multiple Explanationbased learning guided by the Space Fragmenting Algorithm (MESFA), for solving the TBCS problem. The idea is to direct the analytical learning with a specific empirical technology that allows the production of correct concepts regardless of the expressiveness of the concept description language isolated from domain theory. The novelty is that all hypothetical concept definitions within the language are considered as a disjunctive version space. The disjunctive version space is representable by its boundary sets and admits incremental learning with the Space Fragmenting algorithm [4] Space Fragmenting forms a correct, complete and consistent, pure conjunctive or DNF definition of the concept under study.

\section{Representation and Ordering of the Concepts}

The concept definitions in MESFA are expressed in a concept description language $\left(C D L_{D T}\right)$ isolated from supplied domain theory $D T$ :

Definition 1. The concept description language is a finite set of concept definitions: $C D L_{D T}=\{c \mid E \in \mathrm{P}(T S), D T \cup E \vdash c$ such that $D T \cup c \vdash G C\}$, where $\mathrm{P}(T S)$ is powerset of the training set TS and the examples $E$ are subset of TS.

The finiteness of $C D L_{D T}$ is achieved by a proper theorem prover. Between the elements of $C D L_{D T}$ a relation generalized concept ordering holds. The generalized concept ordering is reflexive, anti-symmetric tautologic and transitive:

Definition 2. A concept $c_{2}\left(c_{2} \in C D L_{D T}\right)$ is more general than or equally general to another concept $c_{1}\left(c_{1} \in C D L_{D T}\right)$, written $c_{2} \succeq c_{1}$, in the context of the domain theory $D T$ if and only if the entailment $D T \cup c_{1} \vdash c_{2}$ holds.

It is proven ${ }^{1}$ that all subsets $C$ of $C D L_{D T}$ possess the properties convexity and definiteness [2]. Therefore, the subsets of $C D L_{D T}$ are representable by boundary sets and admit version space learning. MESFA distinguishes by supporting all hypothetical concept definitions in form of a disjunctive version space:

Definition 3. The set $C$ is a disjunctive version space $\left(\mathcal{D V} \mathcal{S}_{S C}\right)$ of the concept under study $S C$ if and only if it consists of all concept definitions $c$ ( $c \in C D L_{D T}$ ) that are not implied by the negative examples $E_{N}$ of $S C$ in context of the domain theory: $C=\left(\mathcal{D V} \mathcal{S}_{S C}\right)$ iff $C=\left\{c \in C D L_{D T} \mid D T \cup E_{N} \nvdash c\right\}$.

With regard to the TBCS problem, definitions 1 and 2 suggest that the disjunctive version space consists of conjunctive concept definitions. Each conjunctive definition summarizes some positive training ( relaxed completeness) while remaining consistent with all of the negative training ( strict consistency). The disjunctive concept definitions are built of conjunctive ones that are: first, not mutually related by generalized concept ordering; and second, the conjunctive definitions together satisfy the completeness requirement.

\footnotetext{
${ }^{1}$ The complete paper with proofs and examples is available from the authors.
} 
The disjunctive version space $\mathcal{D V S}_{S C}$ of the concept under study $S C$ is represented by its maximal and minimal boundary sets $G$ and $S$ :

$\mathcal{D V} \mathcal{S}_{S C}\langle G, S\rangle=\left\{c \in C D L_{D T} \mid \exists s_{i} \in S, \exists g_{i} \in G\right.$ such that $\left.s_{i} \preceq c \preceq g_{i}\right\}$

The set $G$ is the set of maximally general concept definitions in $C D L_{D T}$, which are not implied by the negative examples $E_{N}$ of the concept $S C$ :

$G=\left\{g_{i} \in C D L_{D T} \mid D T \cup E_{N} \nvdash g_{i}\right.$ such that $\left.\neg \exists \mathrm{g} \in C D L_{D T}: g_{i} \prec \mathrm{g}, D T \cup E_{N} \nvdash \mathrm{g}\right\}$

The set $S$ consists of elements $s_{i}$ that are more specific than some corresponding $g_{i}$. Every $s_{i}$ is the minimally specific concept definition implied by all elements of its set $S_{i}$. Every $S_{i}$ is a set of minimally specific concept definitions $s_{i k}$ implied by the positive examples $E_{P i}$ of $S$ C that also imply $g_{i}$ :

$S_{i}=\left\{s_{i k} \in C D L_{D T} \mid D T \cup E_{P i} \vdash s_{i k}, g_{i} \succeq s_{i k}\right.$ such that $\left.\neg \exists \mathrm{s}: \mathrm{s} \prec s_{i k}, D T \cup E_{P i} \vdash \mathrm{s}\right\}$ $S=\left\{s_{i} \in C D L_{D T} \mid D T \cup S_{i} \vdash s_{i}, g_{i} \succeq s_{i}\right.$ such that $\left.\neg \exists \mathrm{s}: \mathrm{s} \prec s_{i}, D T \cup S_{i} \vdash \mathrm{s}\right\}$

For some $g_{i}\left(g_{i} \in G\right)$ the sets of positive examples $E_{P i}$ implying them may be empty. The corresponding $s_{i}$ to these $g_{i}$ are specified with the symbol $\perp$ ( a bottom element implying all elements in the ordered $C D L_{D T}$ ), which is an empty definition that classifies every example as negative.

\section{Scenario for Learning with MESFA}

MESFA is developed employing the Space Fragmenting algorithm. The Space Fragmenting algorithm supports a disjunctive version space of all hypothetical concept definitions of $S C$. It carries out efficient incremental learning by manipulating only the boundary sets $G$ and $S$.

Initialize: $G=\left\{(\text { antecedent of } G C)_{1}\right\} \quad S=\left\{(\perp)_{1}\right\}$

For each next example update $\mathcal{D} \mathcal{V} \mathcal{S}_{S C}\langle G, S\rangle$ into a new one $\mathcal{D} \mathcal{V} \mathcal{S}_{S C}^{\prime}\left\langle G^{\prime}, S^{\prime}\right\rangle$ :

In case of a positive example $e_{p}$ :

$G^{\prime}=G$

$S^{\prime}=\left\{s_{i}^{\prime} \in C D L_{D T} \mid D T \cup S_{i}^{\prime} \vdash s_{i}^{\prime}, g_{i}^{\prime} \succeq s_{i}^{\prime}\right.$ such that $\left.\neg \exists \mathrm{s}: \mathrm{s} \prec s_{i}^{\prime}, D T \cup S_{i}^{\prime} \vdash \mathrm{s}\right\}$ where for each $i$ :

if $g_{i} \succeq e_{p}$ then $S_{i}^{\prime}=\left\{s_{i k}^{\prime} \in C D L_{D T} \mid D T \cup\left\{s_{i}\right\} \cup\left\{e_{p}\right\} \vdash s_{i k}^{\prime}, g_{i}^{\prime} \succeq s_{i k}^{\prime}\right.$ such that $\left.\neg \exists \mathrm{s}: \mathrm{s} \prec s_{i k}^{\prime}, D T \cup\left\{s_{i}\right\} \cup\left\{e_{p}\right\} \vdash \mathrm{s}\right\}$

if $g_{i} \nsucc e_{p}$ then $S_{i}^{\prime}=\left\{s_{i}\right\}$

In case of a negative example $e_{n}$ :

$$
\begin{aligned}
& G^{\prime}=\left\{g_{i}^{\prime} \in C D L_{D T} \mid D T \cup\left\{e_{n}\right\} \nvdash g_{i}^{\prime}, D T \cup\left\{g_{i}^{\prime}\right\} \vdash g_{i}\right. \\
& \text { such that } \left.\neg \exists \mathrm{g} \in C D L_{D T}: g_{i}^{\prime} \prec \mathrm{g}, D T \cup\left\{e_{n}\right\} \nvdash \mathrm{g}, D T \cup\{\mathrm{g}\} \vdash g_{i}\right\} \\
& S^{\prime}=\left\{s_{i}^{\prime} \in C D L_{D T} \mid D T \cup\left\{s_{i}^{\prime}\right\} \vdash s_{i}, s_{i}^{\prime} \preceq g_{i}^{\prime}\right. \\
& \text { such that } \left.\neg \exists \mathrm{s}: \mathrm{s} \succ s_{i}^{\prime}, D T \cup\{\mathrm{s}\} \vdash s_{i}, \mathrm{~s} \preceq g_{i}^{\prime}\right\}
\end{aligned}
$$


MESFA always terminates when the explanations of the positive examples are different from the explanations of the negative ones. This is due to the avoidance of the incompleteness and inconsistency anomalies. Two terminations of the learning process are possible. First, when the elements $g_{i}$ of $G$ are identical with their corresponding $s_{i}$ of $S$, a completely learned definition of the concept $S C$ is generated. Second, when the boundary sets are not focused, an incompletely learned definition of the concept $S C$ is generated. Depending on the power of the set $S$, the resulting concept definition is pure conjunctive or DNF.

\section{Comparisons and Relevance to Other Works}

MESFA and IOE [1] are two approaches to solving the TBCS problem. IOE uses domain theory to build an explanation only from the positive examples and forms definitions of $S C$ by an empirical technique over the explanation. A shortcoming of IOE is that it is not capable of learning from negative examples, and therefore it cannot be applied in real domains where the concepts are DNF rather than pure conjunctive.

MESFA is close to the Hirsh's combination of empirical and analytical learning with IVSM [3]. His integrated approach syntactically processes analytically generalized definitions of the examples. This and the kinds of learned concept definitions suggest that MESFA proposes a more general form of learning.

\section{Conclusion}

A novel approach, called Multiple Explanation-based learning guided by the Space Fragmenting Algorithm (MESFA), was presented. The main advantage of MESFA is that it generates a pure conjunctive or DNF concept definition in an incremental manner in dependence on the provided first order domain theory.

The MESFA approach allows careless design of the domain theory, requires a small number of examples and few computation resources, and may construct a correct definition for several unknown concepts $S C$.

\section{References}

1. N.S.Flann and T.G.Dietterich, A Study of Explanation-Based Methods for Inductive Learning, Machine Learning, 4(2):187-226, 1989.

2. H.Hirsh, Theoretical Underpinnings of Version Spaces, In Proc. of the Twelfth Int. Joint Conf. on Artificial Intelligence,IJCAI-91, pp.665-670, Sydney, Australia,1991.

3. H.Hirsh, Generalizing Version Spaces, Machine Learning, 17(1):5-46, 1994.

4. E.N.Smirnov, Space Fragmenting- A Method For Disjunctive Concept Acquisition, In B.duBoulay and V.Sgurev, eds., Artificial Intelligence V-Methodology, Systems, Applications, pp.97-104, Elsevier Science Publishers, 1992.

5. E.N.Smirnov and N.I.Nikolaev, Multiple Explanation-Based Learning Guided by the Candidate Elimination Algorithm, In P.Jorrand and V.Sgurev, eds., Artificial Intelligence VI- Methodology, Systems, Applications, pp.247-256, World Scientific Publ, Singapore, 1994. 